Research Article

\title{
Follicle-Stimulating Hormone Positively Associates with Metabolic Factors in Perimenopausal Women
}

\author{
Chen Zhang $\mathbb{D})^{1,2}$ Meng Zhao $\mathbb{D},,^{1,2,3}$ Zhengyang Li $\mathbb{D}^{1,2}$ and Yongfeng Song $\mathbb{D}^{1,2,3}$ \\ ${ }^{1}$ Department of Endocrinology, Shandong Provincial Hospital Affiliated to Shandong First Medical University, Jinan 250021, \\ Shandong, China \\ ${ }^{2}$ Shandong Institute of Endocrine \& Metabolic Diseases, Jinan 250021, Shandong, China \\ ${ }^{3}$ Department of Endocrinology, Shandong Provincial Hospital Affiliated to Shandong University, Jinan 250021, Shandong, China
}

Correspondence should be addressed to Yongfeng Song; syf198506@163.com

Received 8 May 2020; Revised 25 October 2020; Accepted 27 October 2020; Published 12 November 2020

Academic Editor: Alberto Ferlin

Copyright (c) 2020 Chen Zhang et al. This is an open access article distributed under the Creative Commons Attribution License, which permits unrestricted use, distribution, and reproduction in any medium, provided the original work is properly cited.

\begin{abstract}
Objective. Menopause is associated with the increased risk of metabolic syndrome (MetS) and cardiovascular (CV) disease. Most studies have focused the postmenopausal women and the relationships among estrogen, androgen, and Mets risk. The main aim of the study was to investigate the Mets risk in perimenopausal women and whether the variation of FSH is associated with metabolic factors. Methods. A single-center cross-sectional retrospective analysis including 154 premenopausal women and 124 perimenopausal women was performed. Results. The prevalence of Mets in the perimenopausal group was much higher than the premenopausal group $(49.19 \%$ vs. $35.71 \%, p=0.023)$. The prevalence of central obesity and NAFLD also increased in the perimenopausal group than in the premenopausal group. We grouped the population by FSH tertiles; compared with women in the lowest tertile, women in the highest tertile had higher age, WC, serum TC, LDL-C, AST, ALT, and creatine levels. The prevalence of hypertriglyceridemia, raised BP and Mets also increased in the highest tertile group. Further, we subdivided the perimenopausal women according to FSH tertiles. Compared with perimenopausal women in the lowest tertile, the prevalence of raised BP significantly increased in the highest tertile. Conclusions. The risk of Mets increased in perimenopausal females than in premenopausal women. And a higher FSH level was associated with higher WC, TG, BPs, and the risk of Mets in perimenopausal women. Elevated FSH level appears to be a risk factor of MetS biomarkers in perimenopausal women.
\end{abstract}

\section{Introduction}

Menopause, characterized by decreased serum estrogen level and increased follicle-stimulating hormone (FSH) level, is a normal part of a woman's life cycle, which has been associated with increased risk of metabolic syndrome (MetS), atherosclerosis, and cardiovascular $(\mathrm{CV})$ disease $[1,2]$. It was reported that the prevalence of MetS in general populations was $20-30 \%[3,4]$ but increased up to $31-69 \%$ after menopause $[5,6]$.

Numerous studies have focused on the relationship between estrogen and metabolic factors, such as lipid, glucose, and obesity [7-9]. Besides the effect of estrogen, some studies paid attention to the increased FSH in menopause women. Sun et al. reported that FSH could stimulate the FSH receptor (FSHR) expressed on the surface of osteoclasts and directly regulated bone mass, resulting in osteoporosis, while estrogen could not correct this effect [10]. Our previous study found that serum FSH is positively correlated with total cholesterol (TC) levels and the prevalence of hypercholesterolemia in women, and blocking FSH inhibits hepatic cholesterol biosynthesis and reduces serum cholesterol [11].

Perimenopause is the transition period from the reproductive to the nonreproductive stage of life, with important changes in their hormone, including FSH and estrogen [12]. Women may experience a number of symptoms associated with the menopausal transition, including vasomotor symptoms, vaginal dryness, sleeping disorders, and the onset of depression [13], and menopause 
transition was also associated with adverse health indicators, such as increased TC and LDL-C levels, increased cardiovascular risk and a greater bone loss/increased bone turnover [14-16]. However, the MetS risks in perimenopause women and the risk factors remain unclear.

In this study, we performed a retrospective study to examine the risk of Mets and the association between FSH and components of MetS among Chinese perimenopausal women. To the best of our knowledge, the current analyses are a part of the few studies focusing on the relationship between FSH and metabolic factors in perimenopausal women.

\section{Materials and Methods}

2.1. Subjects. The cross-sectional study included females aged $\geq 40$ years who participated in an epidemiological investigation performed in Ningyang County in the Shandong Province of China in 2014. All the participants had lived in their current residence for at least 5 years. All participants were instructed to complete a self-reported questionnaire, including detailed questions regarding the menopausal status, provided an overnight fasting blood sample, and underwent a medical examination. Written informed consent was obtained from each participant prior to data collection. This study was approved by the ethics committee of Shandong Provincial Hospital and the procedures were conducted in accordance with the Declaration of Helsinki.

The inclusion criterium was females aged $\geq 40$ years with natural menstrual states. The exclusion criteria were as follows: (1) undergone hormone replacement therapy during the three months prior to the examination; (2) presence of complications or conditions that affect the menopausal status or lipid metabolism, such as ureterectomy, bilateral ovariectomy, premature ovarian failure, history of irregular menstruation during premenopause, pregnancy, lactation, psychiatric illness, acute cardiovascular or cerebrovascular disease, chronic respiratory disease, malignant tumors, or renal dysfunctions; (3) use of medications that affect the natural state of menopause or lipid metabolism, including estrogen, progesterone, androgen, glucocorticoids, statins, fibrates, thyroid hormones, antithyroid drugs or thiazide diuretics, over the preceding three months; (4) subjects with incomplete data.

As a result, 154 premenopausal and 124 perimenopausal female subjects were eligible for inclusion in our study.

2.2. Definition of Variables. The metabolic syndrome was defined according to the Adult Treatment Panel (ATP) III guidelines by the presence of three or more of the five following criteria $[17,18]$ : (1) waist circumference $\geq 80 \mathrm{~cm}$ for women; (2) serum triacylglycerol level $\geq 1.7 \mathrm{mmol} / \mathrm{l}$; (3) HDL-cholesterol level $<1.30 \mathrm{mmol} / 1$ for women; (4) fasting glucose level $\geq 5.6 \mathrm{mmol} / \mathrm{l}$ or use of glucose-lowering drug medication (insulin or oral agents); or (5) systolic blood pressure $\geq 130 \mathrm{mmHg}$ and/or diastolic blood pressure $\geq 85 \mathrm{mmHg}$, and/or use of blood-pressure-lowering medication.
2.3. Data Collection. The determination of the menopausal status was based on the responses to questions regarding menstrual irregularity and amenorrhea in the self-reported questionnaire. Perimenopause was defined as the presence of menses within the past 3 months with a decrease in cycle predictability in the year preceding examination or 3-11 months of amenorrhea.

For the premenopausal participants, venipuncture was scheduled on days $2-5$ of a spontaneous menstrual cycle to evaluate sex hormone levels. For the perimenopausal participants, fasting serum samples were randomly obtained [19]. Blood samples were drawn after 10 hours of overnight fasting. Biomedical analyses, including female sex hormones, serum lipid profiles, plasma glucose level, HbA1c level, serum uric acid (UA) level, and the parameters of hepatic and renal functions, were performed in the clinical laboratory center of Shandong Provincial Hospital.

Trained investigators obtained information on age, sex, and other essential data from the standardized questionnaire [20]. Height was determined to the nearest $1 \mathrm{~cm}$, and weight was determined to the nearest $0.5 \mathrm{~kg}$. Body mass index (BMI) was calculated as the weight in kilograms divided by the square of the height in meters. The waist circumference was measured in centimeters.

2.4. Statistical Analysis. All statistical analyses were performed using SPSS software (25.0 for Windows, SPSS Inc., Chicago, USA). The continuous variables data are presented as mean \pm standard deviation, mean \pm standard error, or median (interquartile range, if nonnormal distribution). Differences between the two groups were compared using the Student's $t$-test or the Mann-Whitney $U$-test (if nonnormal distribution). Differences among multiple groups were compared using one-way analysis of variance (followed by a multiple comparison test for subgroups using Least Significant Difference or Dunnett's T3 tests). All statistical tests were two-tailed, and statistical significance was defined as $p<0.05$.

\section{Results}

3.1. Characteristics of the Study Population. The study population was divided into two groups according to the menstrual status: the perimenopausal group and the premenopausal group. The characteristics of the study population are shown in Table 1 . The whole population contained 154 premenopausal and 124 perimenopausal female subjects. Compared with the premenopausal group, the perimenopausal females were older $(50.06 \pm 5.17$ vs. $46.27 \pm 2.50, p<0.001)$, and the serum FSH and LH levels were elevated significantly while the serum estrogen levels were similar between the two groups. At the same time, the serum progesterone levels were decreased in perimenopausal females.

The body weight and BMI had no difference between the two groups, while the waist circumference was much higher in the perimenopausal group than the premenopausal group $(90.10 \pm 9.40$ vs. $86.92 \pm 9.22, p=0.006)$. The serum TC, 
TABLe 1: The characteristics of the study population.

\begin{tabular}{lccc}
\hline & Premenopause & Perimenopause & \\
& $N=154$ & $N=124$ & $p$ value \\
\hline Age $(\mathrm{ys})$ & $46.27 \pm 2.50$ & $50.06 \pm 5.17$ & $<0.001$ \\
Weight $(\mathrm{kg})$ & $65.92 \pm 10.20$ & $66.41 \pm 10.30$ & 0.697 \\
WC $(\mathrm{cm})$ & $86.92 \pm 9.22$ & $90.10 \pm 9.40$ & 0.006 \\
BMI $\left(\mathrm{kg} / \mathrm{m}^{2}\right)$ & $25.86 \pm 3.52$ & $26.60 \pm 3.77$ & 0.095 \\
SBP $(\mathrm{mmHg})$ & $130.49 \pm 20.31$ & $132.00 \pm 20.78$ & 0.544 \\
DBP $(\mathrm{mmHg})$ & $78.56 \pm 12.90$ & $78.71 \pm 10.86$ & 0.919 \\
FT3 $(\mathrm{pmol} / \mathrm{L})$ & $4.71 \pm 0.48$ & $4.88 \pm 1.15$ & 0.114 \\
FT4 $(\mathrm{pmol} / \mathrm{L})$ & $15.49 \pm 2.02$ & $15.42 \pm 3.50$ & 0.842 \\
TSH $(\mathrm{mIU} / \mathrm{L})$ & $2.95 \pm 1.67$ & $2.72 \pm 1.65$ & 0.270 \\
FSH $(\mathrm{IU} / \mathrm{L})$ & $9.30(6.45 \sim 13.93)$ & $20.87(8.06 \sim 54.87)$ & $<0.001$ \\
LH $(\mathrm{IU} / \mathrm{L})$ & $6.00(4.10 \sim 8.47)$ & $16.64(6.60 \sim 32.32)$ & $<0.001$ \\
E2 $(\mathrm{pg} / \mathrm{ml})$ & $55.12(31.92 \sim 98.29)$ & $61.11(19.16 \sim 146.80)$ & 0.851 \\
PRG $(\mathrm{ng} / \mathrm{ml})$ & $0.52(0.33 \sim 1.74)$ & $0.39(0.20 \sim 0.97)$ & 0.003 \\
FPG $(\mathrm{mmol} / \mathrm{L})$ & $5.78 \pm 1.49$ & $5.81 \pm 1.51$ & 0.906 \\
HbA1c $(\%)$ & $5.75 \pm 0.94$ & $5.81 \pm 0.79$ & 0.604 \\
TC $(\mathrm{mmol} / \mathrm{L})$ & $4.78 \pm 0.85$ & $5.20 \pm 0.95$ & $<0.001$ \\
LDL-C $(\mathrm{mmol} / \mathrm{L})$ & $2.73(2.32 \sim 3.08)$ & $2.97(2.48 \sim 3.45)$ & 0.001 \\
HDL-C $(\mathrm{mmol} / \mathrm{L})$ & $1.39(1.20 \sim 1.55)$ & $1.36(1.18 \sim 1.62)$ & 0.900 \\
TG $(\mathrm{mmol} / \mathrm{L})$ & $0.95(0.78 \sim 1.34)$ & $1.07(0.83 \sim 1.71)$ & 0.023 \\
ALT $(\mathrm{U} / \mathrm{L})$ & $13.85 \pm 5.42$ & $17.02 \pm 6.56$ & $<0.001$ \\
AST $(\mathrm{U} / \mathrm{L})$ & $17.57 \pm 4.86$ & $19.77 \pm 4.85$ & $<0.001$ \\
Crea $(\mu \mathrm{mol} / \mathrm{L})$ & $61.79 \pm 6.16$ & $62.71 \pm 5.36$ & 0.096 \\
UA $(\mu \mathrm{mol} / \mathrm{L})$ & $263.42 \pm 59.82$ & $291.12 \pm 72.90$ & 0.001 \\
\hline All $\mathrm{da}(\mathrm{are}$ expres & &
\end{tabular}

All data are expressed as mean \pm standard deviation or median with interquartile range. Abbreviations: WC: waist circumference; BMI: body mass index; SBP: systolic blood pressure; DBP: diastolic blood pressure; FT3: free triiodothyronine; FT4: free thyroxine; TSH: thyroid stimulating hormone; FSH: follicle stimulating hormone; LH: luteinizing hormone; E2: estrogen; PRG: progesterone; FPG: fasting plasma glucose; TC: total cholesterol; LDL-C: low density lipoprotein cholesterol; HDL-C: high density lipoprotein cholesterol; TG: triglyceride; ALT: Alanine Aminotransferase; AST: Aspartate transaminase; Crea: creatine; UA: uric acid.

LDL-C, TG levels, and the liver enzyme (ALT and AST) and serum UA levels were much higher in perimenopausal females than in premenopause subjects. In addition, the blood pressure (SBP and DBP) levels, HDL-C levels, creatine levels, and thyroid function (FT3, FT4, TSH) had no significant difference between the two groups.

3.2. Risk of Mets Increased in Perimenopausal Females. As shown in Table 2, by definition, the overall prevalence of Mets in the whole study population was $41.73 \%$. In the premenopausal group, $35.71 \%$ burdened from the Mets, while in the perimenopausal group, it was up to $49.19 \%$, and the difference between the two groups was statistically significant $(p=0.023)$.

Further, we also observed the difference of different metabolic components between the two groups. Only the prevalence of central obesity increased in the perimenopausal group, while the prevalence of hypertriglyceridemia, raised FPG, hypertension, and low HDL had no difference between the two groups. Nonalcoholic fatty liver disease (NAFLD) often cooccurred with one or more MetS-associated phenotypes and they shared common pathogenic mechanisms [21]. We found that the prevalence of NAFLD also increased in the perimenopausal group than the premenopausal group ( $45.97 \%$ vs. $30.17 \%, p=0.012)$.
3.3. FSH is Positively Associated with Risks of MetS Biomarkers. The serum FSH levels increased while the serum estrogen levels had no significant change in the perimenopausal group, so we grouped the population by FSH tertiles. The tertile ranges of FSH in the population were $<8.55$ (Q1), 8.55 19.11 (Q2), > 19.11 IU/L (Q3). As shown in Table 3, when we compared with women in the lowest tertile (Q1), women in the highest tertile (Q3) had higher age, WC, serum TC, LDL-C, AST, ALT, and creatine levels. The serum estrogen and progesterone levels were expected to decrease with FSH increased.

We also observed the prevalence of MetS biomarkers. Overall, compared with women in the lowest tertile (Q1), the prevalence of hypertriglyceridemia, raised $\mathrm{BP}$, and Mets increased in the highest tertile (Q3) group. However, the prevalence of raised FPG and low HDL had no difference (Table 4).

Further, we subdivided the perimenopausal women into three groups according to FSH tertiles $(\mathrm{Q} 1:<10.38$, Q2: 10.38 39.05, Q3: >39.05 IU/L) and examined the association of FSH with risks of MetS biomarkers in perimenopausal women. Compared with women in the lowest tertile (Q1), the prevalence of raised BP significantly increased in the highest tertile (Q3). The risk of Mets was higher in Q3 than in Q1, and the $p$ value was close to the significance $(p=0.090)$. However, the prevalence of central obesity raised TG and FPG, and low HDL had no difference (Table 5). We noticed that the sample size was relatively small after classified into three groups $(n=40)$, and thus the statistic power might not be enough to detect a moderate effect.

\section{Discussion}

In this study, we found that the risk of Mets increased in perimenopausal females than in premenopausal women. A higher FSH level was associated with higher WC, TG, BPs, and the risk of Mets in perimenopausal women.

Midlife women have a high risk of Mets, which was reported that they had a one-in-three chance of developing MetS that predisposes them to T2DM and CVD [22, 23]. Midlife women usually underwent menopausal transition (MT) [24]. And the prevalence of the MetS increased from premenopause to postmenopause in women, independent of age [24]. Perimenopause is an intermediate stage between premenopause and postmenopause. This period corresponds to the final depletion of oocytes and can last up to 10 years, when women may experience important changes in their hormone [25]. However, The Mets risk in perimenopausal women is still unclear. To the best of our knowledge, this is one of the few studies to detect the association between FSH level and MetS biomarkers of normal perimenopausal women in a population-based investigation.

A hallmark of the menopausal transition is the dramatic reduction in estradiol levels, and with this reduction, there is a progressive shift toward androgen dominance in the hormonal milieu [26, 27]. Most of the previous studies have focused on the effects of estrogen and androgen fluctuation on Mets and CVD risk [28, 29]. Besides the reduction of estrogen, 
TABLE 2: The risk of Mets increased in perimenopausal females.

\begin{tabular}{lccc}
\hline Components of MetS, $n(\%)$ & Premenopause $n=154$ & Peri-menopause $n=124$ & Total $n=278$ \\
\hline Central obesity & $116(77.33)$ & $106(88.33)$ & $222(82.22)$ \\
Raised TG & $25(16.23)$ & $31(25.00)$ & $56(20.14)$ \\
Low HDL-C & $62(40.26)$ & $55(44.35)$ & $117(42.09)$ \\
Raised FPG & $65(42.21)$ & $57(45.97)$ & $122(43.88)$ \\
Raised BP & $69(46.00)$ & $67(54.03)$ & 0.070 \\
MetS & $55(35.71)$ & $61(49.19)$ & 0.492 \\
NAFLD & $35(30.17)$ & $57(45.97)$ & 0.1330 \\
\hline
\end{tabular}

Data are expressed as number with proportion for categorical variables. Pearson's chi-squared test was used in the comparison of dichotomous variables.

TABLE 3: The characteristics of the study population with different FSH levels.

\begin{tabular}{|c|c|c|c|c|}
\hline & & Serum FSH tertiles & & \\
\hline & Q1 & Q2 & Q3 & $P$ value \\
\hline Age (ys) & $47.82 \pm 5.18$ & $46.65 \pm 2.78$ & $50.13 \pm 4.62 *$ & $<0.001$ \\
\hline Weight (kg) & $66.39 \pm 10.09$ & $64.43 \pm 10.03$ & $67.33 \pm 10.48$ & 0.199 \\
\hline $\mathrm{WC}(\mathrm{cm})$ & $88.57 \pm 8.74$ & $86.12 \pm 9.39$ & $90.73 \pm 8.92 \$ \$$ & 0.008 \\
\hline BMI $\left(\mathrm{kg} / \mathrm{m}^{2}\right)$ & $26.19 \pm 3.54$ & $25.52 \pm 3.75$ & $26.74 \pm 3.56 \$$ & 0.110 \\
\hline SBP (mmHg) & $132.09 \pm 23.02$ & $127.87 \pm 18.01$ & $134.63 \pm 21.80 \$$ & 0.128 \\
\hline $\mathrm{DBP}(\mathrm{mmHg})$ & $78.11 \pm 13.63$ & $78.15 \pm 11.68$ & $79.17 \pm 11.06$ & 0.826 \\
\hline $\mathrm{E} 2(\mathrm{pg} / \mathrm{ml})$ & $120.40(63.20,161.70)$ & $60.11(34.18,95.86) *$ & $22.16(13.08,46.36) * * \$$ & $<0.001$ \\
\hline PRG (ng/ml) & $0.95(0.39,9.46)$ & $0.54(0.32,1.56) *$ & $0.30(0.16,0.47) * * \$$ & $<0.001$ \\
\hline $\mathrm{FPG}(\mathrm{mmol} / \mathrm{L})$ & $5.59 \pm 1.01$ & $5.79 \pm 1.49$ & $5.96 \pm 1.80$ & 0.282 \\
\hline HbAlc (\%) & $5.65 \pm 0.54$ & $5.76 \pm 0.93$ & $5.91 \pm 1.06$ & 0.171 \\
\hline $\mathrm{TC}(\mathrm{mmol} / \mathrm{L})$ & $4.82 \pm 0.82$ & $4.89 \pm 0.91$ & $5.29 \pm 1.04 * * \$ \$$ & 0.003 \\
\hline LDL-C (mmol/L) & $2.76 \pm 0.69$ & $2.79 \pm 0.61$ & $3.07 \pm 0.80 * * \$$ & 0.014 \\
\hline HDL-C (mmol/L) & $1.38 \pm 0.26$ & $1.40 \pm 0.23$ & $1.41 \pm 0.34$ & 0.771 \\
\hline $\mathrm{TG}(\mathrm{mmol} / \mathrm{L})$ & $0.97(0.72,1.38)$ & $0.95(0.77,1.33)$ & $1.09(0.84,1.77)$ & 0.597 \\
\hline $\operatorname{ALT}(\mathrm{U} / \mathrm{L})$ & $14.57 \pm 5.70$ & $14.49 \pm 6.39$ & $16.99 \pm 6.23 * \$$ & 0.016 \\
\hline $\mathrm{AST}(\mathrm{U} / \mathrm{L})$ & $18.23 \pm 4.05$ & $17.82 \pm 4.94$ & $20.10 \pm 5.04 * \$ \$$ & 0.006 \\
\hline Crea $(\mu \mathrm{mol} / \mathrm{L})$ & $60.46 \pm 5.56$ & $62.24 \pm 4.76$ & $63.82 \pm 6.73 *$ & 0.001 \\
\hline $\mathrm{UA}(\mu \mathrm{mol} / \mathrm{L})$ & $278.21 \pm 60.35$ & $271.37 \pm 77.60$ & $290.54 \pm 65.67$ & 0.245 \\
\hline
\end{tabular}

All data are expressed as mean \pm standard deviation or median with interquartile range. The tertile ranges of FSH in the population were $<8.55$ (Q1), 8.55 19.11 (Q2), $>19.11 \mathrm{IU} / \mathrm{L}(\mathrm{Q} 3) . * p<0.05, * p<0.01$ vs Q1 group; $\$ p<0.05, \$ \$ p<0.01$ vs. Q2 gro.

TABLE 4: FSH is positively associated with risks of MetS biomarkers.

\begin{tabular}{lcccc}
\hline $\begin{array}{l}\text { Components of } \\
\text { MetS, } n(\%)\end{array}$ & Q1 $n=79$ & Q2 $n=79$ & Q3 $n=78$ & $\begin{array}{c}p \\
\text { value }\end{array}$ \\
\hline Central obesity & $66(86.84)$ & $57(73.08)^{*}$ & $68(90.67)^{\$ \$}$ & 0.009 \\
Raised TG & $14(17.72)$ & $9(11.39)$ & $21(26.92)^{\$}$ & 0.043 \\
Low HDL-C & $31(39.24)$ & $31(39.24)$ & $34(43.59)$ & 0.815 \\
Raised FPG & $32(40.51)$ & $34(43.04)$ & $38(48.72)$ & 0.570 \\
Raised BP & $33(41.77)$ & $36(45.57)$ & $49(62.82)^{* * \$}$ & 0.019 \\
Mets & $27(34.17)$ & $26(32.91)$ & $38(48.72)^{*}$ & 0.043 \\
\hline
\end{tabular}

Data are expressed as number with proportion for categorical variables. Pearson chi-squared test was used in the comparison for dichotomous variables. $* p<0.05, * p<0.01$ vs Q1 group; $\$ p<0.05, \$ \$ p<0.01$ vs. Q2 group.

FSH levels increased sharply which was earlier than the estrogen reduction [25]. Strong correlations were found to exist between rising serum FSH levels and osteoporosis and obesity after menopause, and blocking FSH reduced body fat levels and increased the bone density in menopause [10, 30, 31]. Furthermore, postmenopausal women with a higher serum FSH ( $\geq 78.3$ IU/L) had higher serum TC and LDL-C levels than those with relatively lower FSH levels (40-78.3 IU/L) [32], and FSH was positively associated with serum TC and
TABLE 5: The risks of MetS biomarkers according to FSH tertiles in perimenopausal women.

\begin{tabular}{lcccc}
\hline $\begin{array}{l}\text { Components of } \\
\text { MetS, } n(\%)\end{array}$ & Q1 $n=40$ & Q2 $n=41$ & Q3 $n=40$ & $\begin{array}{c}p \\
\text { value }\end{array}$ \\
\hline Central obesity & $34(87.18)$ & $32(84.21)$ & $38(92.50)$ & 0.490 \\
Raised TG & $8(20.00)$ & $9(21.95)$ & $12(30.00)$ & 0.539 \\
Low HDL-C & $20(50.00)$ & $15(36.59)$ & $18(45.00)$ & 0.469 \\
Raised FPG & $13(32.50)$ & $21(51.22)$ & $21(52.50)$ & 0.131 \\
Raised BP & $18(45.00)$ & $19(46.34)$ & $29(72.50)^{*}$ & 0.020 \\
Mets & $17(43.69)$ & $18(43.90)$ & $26(65.00)$ & 0.090 \\
\hline
\end{tabular}

Data are expressed as number with proportion for categorical variables. Pearson chi-squared test was used in the comparison for dichotomous variables. $* p<0.05, * p<0.01$ vs $\mathrm{Q} 1$ group; $\$ p<0.05, \$ \$ p<0.01$ vs. Q2 group.

LDL-C levels, which was independent of age and estrogen [11]. It was reported that even with regular menstruation, women with FSH $\geq 7$ IU/L had higher TC and LDL levels than those with FSH $<7$ IU/L [33]. All these results suggested that FSH was also of great importance in metabolic regulation. Likewise, FSH receptor polymorphism seemed to play an important role in increased Mets risk in women. Cannarella $\mathrm{R}$ et al. have demonstrated that FSHR gene polymorphism, 
rs6166, significantly influences glucose metabolism [34]. Moreover, it was reported that women having activated FSHR polymorphisms also have lower bone mass and higher bone turnover [35]. In this study, we also found that a higher FSH level was associated with higher WC, TG, BPs, and the risk of Mets in perimenopausal women.

Central obesity was the most frequent component of Mets for midlife women [2]. In our study, we also found that there was no difference in weight and BMI between premenopausal and perimenopausal women, while the waist circumference increased in perimenopausal women, and the prevalence of central obesity was highest than other Mets components. The WC levels increased with the FSH level increase. These associations might be explained by the involvement of FSH in fat accumulation and redistribution in humans. FSH was found to bind to FSHR in adipocytes and promote excessive lipid biosynthesis and lipid droplet formation in visceral adipose tissue through the $\mathrm{G} \alpha \mathrm{i} / \mathrm{Ca}^{2+} / \mathrm{CREB}$ pathway [36]. Moreover, blocking FSH activated thermogenic adipose tissue and reduced adiposity [31]. A previous study also found that $(\log )$ FSH change was positively correlated with $(\log )$ fat mass change after menopause [37]. These results suggested that central obesity might be the pathological basis for metabolic disturbance in perimenopausal women.

Some studies also reported the associations between FSH and other Mets components in postmenopausal women. SBP and DBP increased by 6.11 and $3.54 \mathrm{mmHg}$ after menopause, respectively [38]. One study concluded that the increase in FSH was related to an increase in blood pressure in postmenopausal women [39]. In that study, the intracellular mechanism of FSH promoting catecholamine synthesis in PC12 and rat adrenal medulla chromaffin cells was proposed. The SPECT-China study found that low FSH was associated with prediabetes and diabetes in postmenopausal women [40]. Sun et al. also reported that postmenopausal women in the lower FSH level had higher FBG [41]. However, in our study, we did not find any difference for FPG and blood pressure in premenopausal and perimenopausal women. While FSH increased, the prevalence of raised $\mathrm{BP}$ also increased. The inconsistent results may be due to the different study population. We used perimenopausal women while other studies used postmenopausal subjects. In contrast, the metabolic disturbance was much more serious in postmenopausal subjects than perimenopausal women.

Our study also has some limitations. The main limitation was the small samples. We only enrolled 154 premenopausal women and 124 perimenopausal women. Some phenomenon may be masked by the small samples. Second, this was a retrospective study that cannot establish causal relationships. A large-scale, randomized, prospective study would be a better study design to investigate the relationship between FSH and MetS, as well as MetS biomarkers. Third, we used a self-reported questionnaire to determine menopausal status but did not have the Anti-Müllerian hormone $(\mathrm{AMH})$, which is a reliable indicator of ovarian reserve and menopausal status [42]. Fourth, the lack of insulin levels and HOMAi limited our further explorations of their associations with FSH levels.
In conclusion, the risk of Mets increased in perimenopausal females. A higher FSH level appears to be a risk factor of MetS biomarkers in perimenopausal women. However, further clinical and mechanistic studies are needed to explore the effect of FSH on metabolism disturbance.

\section{Data Availability}

The data sets used and analyzed during the current study could be made available upon reasonable request to the corresponding author.

\section{Ethical Approval}

All procedures performed in studies involving human participants were in accordance with the ethical standards of the ethics committee of Shandong Provincial Hospital affiliated to Shandong First Medical University and with the 1964 Helsinki Declaration and its later amendments or comparable ethical standards.

\section{Consent}

Informed consent was obtained from all individual participants included in the study

\section{Conflicts of Interest}

The authors declare that they have no conflicts of interest.

\section{Acknowledgments}

This study was supported by the National Natural Science Foundation (81922016 and 81870607), Shandong Provincial Natural Science Foundation (ZR2019JQ25), and National Key R\&D Program of China (2017YFC0908900).

\section{References}

[1] K. L. Marlatt, L. M. Redman, R. A. Beyl et al., "Racial differences in body composition and cardiometabolic risk during the menopause transition: a prospective, observational cohort study," American Journal of Obstetrics and Gynecology, vol. 222, no. 4, pp. 365.e1-365.e18, 2019.

[2] E. Ward, E. B. Gold, W. O. Johnson et al., "Patterns of cardiometabolic health as midlife women transition to menopause: a prospective multiethnic study," The Journal of Clinical Endocrinology \& Metabolism, vol. 104, no. 5, pp. 1404-1412, 2019.

[3] B. Xi, D. He, Y. Hu, and D. Zhou, "Prevalence of metabolic syndrome and its influencing factors among the Chinese adults: the China health and nutrition survey in 2009," Preventive Medicine, vol. 57, no. 6, pp. 867-871, 2013.

[4] J. K. Vishram, A. Borglykke, A. H. Andreasen et al., "Impact of age and gender on the prevalence and prognostic importance of the metabolic syndrome and its components in Europeans. the MORGAM prospective cohort project," PLoS One, vol. 9, Article ID e107294, 2014.

[5] A. Stefanska, K. Bergmann, and G. Sypniewska, "Metabolic syndrome and menopause," Advances in Clinical Chemistry, vol. 72, pp. 1-75, 2015. 
[6] Y.-W. Park, S. Zhu, L. Palaniappan, S. Heshka, M. R. Carnethon, and S. B. Heymsfield, "The metabolic syndrome," Archives of Internal Medicine, vol. 163, no. 4, pp. 427-436, 2003.

[7] S. A. Litwak, J. L. Wilson, W. Chen et al., "Estradiol prevents fat accumulation and overcomes leptin resistance in female high-fat diet mice," Endocrinology, vol. 155, no. 11, pp. 4447-4460, 2014.

[8] N. Wenger, "Clinical characteristics of coronary heart disease in women: emphasis on gender differences," Cardiovascular Research, vol. 53, no. 3, pp. 558-567, 2002.

[9] F. Mauvais-Jarvis, J. E. Manson, J. C. Stevenson, and V. A. Fonseca, "Menopausal hormone therapy and type 2 diabetes prevention: evidence, mechanisms, and clinical implications," Endocrine Reviews, vol. 38, no. 3, pp. 173-188, 2017.

[10] L. Sun, Y. Peng, A. C. Sharrow et al., "FSH directly regulates bone mass," Cell, vol. 125, no. 2, pp. 247-260, 2006.

[11] Y. Guo, M. Zhao, T. Bo et al., "Blocking FSH inhibits hepatic cholesterol biosynthesis and reduces serum cholesterol," Cell Research, vol. 29, no. 2, pp. 151-166, 2019.

[12] R. O. G. Carolino, P. T. Barros, B. Kalil, and J. AnselmoFranci, "Endocrine profile of the VCD-induced perimenopausal model rat," PLoS One, vol. 14, Article ID e0226874, 2019.

[13] E. B. Gold, A. Colvin, N. Avis et al., "Longitudinal analysis of the association between vasomotor symptoms and race/ethnicity across the menopausal transition: study of women's health across the nation," American Journal of Public Health, vol. 96, no. 7, pp. 1226-1235, 2006.

[14] C. J. Crandall, C.-H. Tseng, S. L. Crawford et al., “Association of menopausal vasomotor symptoms with increased bone turnover during the menopausal transition," Journal of Bone and Mineral Research, vol. 26, no. 4, pp. 840-849, 2011.

[15] R. C. Thurston, K. Sutton-Tyrrell, S. A. Everson-Rose, R. Hess, and K. A. Matthews, "Hot flashes and subclinical cardiovascular disease," Circulation, vol. 118, no. 12, pp. 1234-1240, 2008.

[16] C. A. Derby, S. L. Crawford, R. C. Pasternak, M. Sowers, B. Sternfeld, and K. A. Matthews, "Lipid changes during the menopause transition in relation to age and weight: the study of women's health across the nation," American Journal of Epidemiology, vol. 169, no. 11, pp. 1352-1361, 2009.

[17] J. D. van der Berg, C. D. A. Stehouwer, H. Bosma et al., "Associations of total amount and patterns of sedentary behaviour with type 2 diabetes and the metabolic syndrome: the Maastricht study," Diabetologia, vol. 59, no. 4, pp. 709-718, 2016.

[18] K. G. M. M. Alberti, R. H. Eckel, S. M. Grundy et al., "Harmonizing the metabolic syndrome," Circulation, vol. 120, no. 16, pp. 1640-1645, 2009.

[19] N. Nagaraj, K. A. Matthews, K. J. Shields, E. Barinas-Mitchell, M. J. Budoff, and S. R. El Khoudary, "Complement proteins and arterial calcification in middle aged women: cross-sectional effect of cardiovascular fat. the SWAN cardiovascular fat ancillary study," Atherosclerosis, vol. 243, no. 2, pp. 533-539, 2015.

[20] R. V. Luepker, A. Evans, P. McKeigue, and K. Srinath, Cardiovascular Survey Methods, World Health Organization, Geneva, Switzerland, 3 edition, 2004.

[21] S. Sookoian and C. J. Pirola, "Nonalcoholic fatty liver disease and metabolic syndrome: shared genetic basis of pathogenesis," Hepatology, vol. 64, no. 5, pp. 1417-1420, 2016.
[22] E. S. Ford, W. H. Giles, and W. H. Dietz, "Prevalence of the metabolic syndrome among US adults," Journal of the American Medical Association, vol. 287, no. 3, pp. 356-359, 2002.

[23] E. S. Ford, C. Li, and G. Zhao, "Prevalence and correlates of metabolic syndrome based on a harmonious definition among adults in the US," Journal of Diabetes, vol. 2, no. 3, pp. 180-193, 2010.

[24] I. Janssen, L. H. Powell, S. Crawford, B. Lasley, and K. SuttonTyrrell, "Menopause and the metabolic syndrome," Archives of Internal Medicine, vol. 168, pp. 1568-1575, 2008.

[25] N. Santoro and J. F. Randolph Jr, "Reproductive hormones and the menopause transition," Obstetrics and Gynecology Clinics of North America, vol. 38, pp. 455-466, 2011.

[26] H. G. Burger, E. C. Dudley, J. Cui, L. Dennerstein, and J. L. Hopper, "A prospective longitudinal study of serum testosterone, dehydroepiandrosterone sulfate, and sex hormone-binding globulin levels through the menopause transition," The Journal of Clinical Endocrinology and Metabolism, vol. 85, pp. 2832-2838, 2000.

[27] B. L. Lasley, N. Santoro, J. F. Randolf et al., "The relationship of circulating dehydroepiandrosterone, testosterone, and estradiol to stages of the menopausal transition and ethnicity," The Journal of Clinical Endocrinology and Metabolism, vol. 87, pp. 3760-3767, 2002.

[28] K. A. Do, A. Green, J. R. Guthrie, E. C. Dudley, H. G. Burger, and L. Dennerstein, "Longitudinal study of risk factors for coronary heart disease across the menopausal transition," Americal Journal of Epidemiology, vol. 151, pp. 584-593, 2000.

[29] J. R. Guthrie, J. R. Taffe, P. Lehert, H. G. Burger, and L. Dennerstein, "Association between hormonal changes at menopause and the risk of a coronary event: a longitudinal study," Menopause, vol. 11, pp. 315-322, 2004.

[30] L. L. Zhu, H. Blair, J. Cao et al., "Blocking antibody to the $\beta$-subunit of FSH prevents bone loss by inhibiting bone resorption and stimulating bone synthesis," Proceedings of the National Academy of Sciences, vol. 109, pp. 14574-14579, 2012.

[31] P. Liu, Y. Ji, T. Yuen et al., "Blocking FSH induces thermogenic adipose tissue and reduces body fat," Nature, vol. 546, pp. 107-112, 2017.

[32] Y. Song, E.-S. Wang, L.-L. Xing et al., "Follicle-stimulating hormone induces postmenopausal dyslipidemia through inhibiting hepatic cholesterol metabolism," The Journal of Clinical Endocrinology \& Metabolism, vol. 101, pp. 254-263, 2016.

[33] M. C. Chu, K. M. Rath, J. Huie, and H. S. Taylor, "Elevated basal FSH in normal cycling women is associated with unfavourable lipid levels and increased cardiovascular risk," Human Reproduction, vol. 18, pp. 1570-1573, 2003.

[34] R. Cannarella, N. Musso, R. A. Condorelli et al., "The 2039 A/G FSH receptor gene polymorphism influences glucose metabolism in healthy men," Endocrine, 2020.

[35] D. Rendina, F. Gianfrancesco, G. De Filippo et al., "FSHR gene polymorphisms influence bone mineral density and bone turnover in postmenopausal women," European Journal of Endocrinology, vol. 163, pp. 165-172, 2010.

[36] X. M. Liu, H. C. Chan, G. L. Ding et al., "FSH regulates fat accumulation and redistribution in aging through the Gai/ $\mathrm{Ca}(2+) / C R E B$ pathway," Aging Cell, vol. 14, pp. 409-420, 2015.

[37] M. Sowers, H. Zheng, K. Tomey et al., "Changes in body composition in women over six years at midlife: ovarian and 
chronological aging," The Journal of Clinical Endocrinology and Metabolism, vol. 92, pp. 895-901, 2007.

[38] D. Pu, R. Tan, Q. Yu, and J. Wu, "Metabolic syndrome in menopause and associated factors: a meta-analysis," Climacteric, vol. 20, pp. 583-591, 2017.

[39] J. Yang, Elevated follicle-stimulating hormone induces postmenopausal vascular dysfunction by promoting adrenal catecholamine synthesis, $\mathrm{PhD}$ thesis, Zhejiang University, Zhejiang, China, 2014.

[40] N. Wang, L. Kuang, B. Han et al., "Follicle-stimulating hormone associates with prediabetes and diabetes in postmenopausal women," Acta Diabetologica, vol. 53, pp. 227236, 2016

[41] L. Gao, X. Wu, X. Zhu, Q. Jin, Q. Ma, and A. Sun, "Folliclestimulating hormone associates with metabolic factors in postmenopausal women," Gynecoloical Endocrinology, vol. 34, pp. 1035-1038, 2018.

[42] A. Kruszyńska and J. Słowińska-Srzednicka, "Anti-Müllerian hormone (AMH) as a good predictor of time of menopause," Przeglad Menopauzalny=Menopause Review, vol. 16, pp. 4750, 2017. 